\title{
On Random Sections of the Cube*
}

\section{Y. Lonke ${ }^{\dagger}$}

Institute of Mathematics,

The Hebrew University of Jerusalem,

Jerusalem 91904, Israel

\begin{abstract}
Let $f(j, k, n)$ denote the expected number of $j$-faces of a random $k$-section of the $n$-cube. A formula for $f(0, k, n)$ is presented, and, for $j \geq 1$, a lower bound for $f(j, k, n)$ is derived, which implies a precise asymptotic formula for $f(n-m, n-l, n)$ when $1 \leq l<m$ are fixed integers and $n \rightarrow \infty$.
\end{abstract}

\section{Introduction}

The principal object in this paper is the expected number of $j$-dimensional faces (in short, $j$-faces) of a random $k$-dimensional central section (in short, $k$-section) of the $n$-cube $B_{\infty}^{n}=[-1,1]^{n}$ in $\mathbb{R}^{n}$. We denote this number by $f(j, k, n)$. The normalized rotation invariant measure on the set $G_{n, k}$ of all $k$-dimensional subspaces of $\mathbb{R}^{n}$ provides the probabilistic framework.

Section 2 contains a calculation of the expected number of vertices of a random $k$-section of the $n$-cube. The result is

$$
f(0, k, n)=2^{k}\left(\begin{array}{l}
n \\
k
\end{array}\right) \sqrt{\frac{2 k}{\pi}} \int_{0}^{\infty} e^{-k t^{2} / 2} \gamma_{n-k}\left(t B_{\infty}^{n-k}\right) d t
$$

where $\gamma_{n-k}$ denotes the $(n-k)$-dimensional Gaussian probability measure.

\footnotetext{
* This work was supported in part by the US-Israel Binational Science Foundation. This work forms part of a dissertation prepared under the direction of Professor Joram Lindenstrauss, to whom I am greatly indebted for much valuable advice.

$\dagger$ Current address: Department of Mathematics, Case Western Reserve University, Cleveland, OH 441067058,USA.jr116@po.cwru.edu.
} 
In Section 3 we derive a lower bound for $f(j, k, n)$ for every $1 \leq j<k<n$. The main result is

$$
\frac{f(0, k-j, n)}{f(j, k, n)}<\sqrt{\frac{2}{\pi}}\left(\frac{j(k-j)}{n-k+j}\right)^{1 / 2} \int_{0}^{\infty} \exp \left(-\frac{j(k-j)}{n-k+j} t^{2} / 2\right) \gamma_{j}\left(t B_{\infty}^{j}\right) d t .
$$

The lower bound for $f(j, k, n)$ derived from this inequality, combined with (1), leads in some cases to asymptotically best possible results. For example, in Section 3 we deduce from it the following asymptotic formula, for fixed integers $1 \leq l<m$ :

$$
f(n-m, n-l, n) \backsim \frac{(2 n)^{m-l}}{(m-l) !} \quad \text { as } \quad n \rightarrow \infty .
$$

The notation $a_{n} \sim b_{n}$ means $a_{n} / b_{n} \rightarrow 1$ as $n \rightarrow \infty$. Formula (2) can be interpreted as follows: the probability that a random fixed-codimensional subspace of $\mathbb{R}^{n}$ intersects a fixed-codimensional face of the $n$-cube, tends to 1 as $n \rightarrow \infty$. Formula (2) itself follows also from the work of Affentranger and Schneider. (See Remark 1 of Section 3 below.) In [1], they found a formula for the expected number $\mathbf{E}\left(f_{j}\left(\Pi_{k} P\right)\right)$ of $j$-faces of an orthogonal projection of an $n$-polytope $P$ onto a $k$-dimensional random subspace. Formula (5) of [1] reads as follows:

$$
\mathbf{E}\left(f_{j}\left(\Pi_{k} P\right)\right)=f_{j}(P)-2 \sum_{s \geq 0} \sum_{F \in \mathcal{F}_{j}(P)} \sum_{G \in \mathcal{F}_{k+1+2 s}(P)} \beta(F, G) \gamma(G, P) .
$$

Here $\mathcal{F}_{j}(P)$ denotes the set of $k$-faces of $P$, and $f_{j}(P)=\operatorname{card} \mathcal{F}_{j}(P) . \beta(F, G)$ denotes the internal angle [8, p. 297], of the face $G$ at its face $F$, and $\gamma(G, P)$ - the external angle [8, p. 308], of $P$ at its face $G$. It is shown in [1] that (3) implies that if $0 \leq j<k$ are fixed integers, then, as $n \rightarrow \infty$,

$$
\mathbf{E}\left(f_{j}\left(\Pi_{k} T^{n}\right)\right) \sim \frac{2^{k}}{\sqrt{k}}\left(\begin{array}{c}
k \\
j+1
\end{array}\right) \beta\left(T^{j}, T^{k-1}\right)(\pi \log n)^{(k-1) / 2} .
$$

Here $T^{n}$ stands for the regular $n$-simplex.

In a very recent work [5], Böröczky, Jr., and Henk showed that (3) implies the same asymptotic formula (4) also for $\mathbf{E}\left(f_{j}\left(\Pi_{k} B_{1}^{n}\right)\right)$, where $B_{1}^{n}$ is the regular cross-polytope. In addition, they found an asymptotic formula for the internal angles $\beta\left(T^{j}, T^{k-1}\right)$, when $k / j^{2} \rightarrow \infty$. Therefore if $j$ is fixed, $k$ is much larger than $j^{2}$ and $n$ much larger than $k$, then explicit estimates for $\mathbf{E}\left(f_{j}\left(\Pi_{k} B_{1}^{n}\right)\right)$ are available. See [5] for more details. Explicit asymptotic formulas for $\mathbf{E}\left(f_{j}\left(\Pi_{k} T^{n}\right)\right)$ were established independently by Vershik and Sporyshev [10], when $j, k$ are both proportional to $n$ and $n \rightarrow \infty$.

A simple duality argument shows that

$$
\mathbf{E}\left(f_{j}\left(\Pi_{k} B_{1}^{n}\right)\right)=f(k-j-1, k, n) .
$$

Choose $j=k-1$ in (4). Applying the result for $B_{1}^{n}$, one has

$$
f(0, k, n)=\mathbf{E}\left(f_{k-1}\left(\Pi_{k} B_{1}^{n}\right)\right) \sim \frac{2^{k}}{\sqrt{k}}(\pi \log n)^{(k-1) / 2} \quad \text { as } \quad n \rightarrow \infty .
$$

The last asymptotic formula follows also from (1). In fact, if $\left\{g_{i}\right\}_{i=1}^{m}$ are independent $N(0,1)$ (that is, with mean 0 and variance 1$)$ Gaussian variables, then $\gamma_{m}\left(t B_{\infty}^{m}\right)$ coincides 
with the probability of the event $\left\{\max _{1 \leq i \leq m}\left|g_{i}\right| \leq t\right\}$. This probabilistic interpretation allows a straightforward evaluation of the asymptotic behavior of the integral in (1), when $k$ is fixed and $n \rightarrow \infty$.

Formula (1) also yields information about $f(0, k, n)$ for $k$ not necessarily fixed. For example, if $k=n-1$, then the integral in (1) can be computed and the result is

$$
f(0, n-1, n)=\frac{2^{n} n}{\pi} \arctan \frac{1}{\sqrt{n-1}} \sim \frac{2^{n} \sqrt{n}}{\pi} .
$$

Particular values of the last formula were computed numerically in Table 2 of [5]. For the expected number of vertices of random sections of fixed codimension, we have the following inequality, which is a consequence of (1):

$$
f(0, n-d, n) \geq\left(\begin{array}{l}
n \\
d
\end{array}\right) 2^{n}\left(\frac{1}{\pi} \arctan \frac{1}{\sqrt{n-d}}\right)^{d} \quad(d \geq 1) .
$$

Equality holds for $d=1$.

To obtain a lower bound for $f(j, k, n)$, it turns out that it is useful to know an estimate for the Gaussian measure of a cone generated by a section of a face of a cube. In Section 3 we find such an estimate, by modifying Ball's calculation of the maximal volume of a cube-section, based on Brascamp-Lieb's inequality [2].

Dvoretzky's theorem on almost Euclidean sections asserts that there exists a function $k(\varepsilon, n) \geq 1$, tending to infinity as $n \rightarrow \infty$ for each fixed $\varepsilon>0$, such that if $K$ is an $n$-dimensional centrally symmetric convex body (that is, a convex compact set in $\mathbb{R}^{n}$ with nonempty interior, satisfying $K=-K)$, and $\varepsilon>0$, then for each $1 \leq k \leq k(\varepsilon, n)$ there exists a $k$-dimensional subspace $X$, and a linear automorphism $T$ of $X$ for which

$$
X \cap B_{2}^{n} \subset T(X \cap K) \subset(1+\varepsilon)\left(X \cap B_{2}^{n}\right),
$$

where $B_{2}^{n}$ denotes the Euclidean unit ball. The proof of Dvoretzky's theorem in [6] shows that $k(\varepsilon, n) \geq c \varepsilon^{2}|\log \varepsilon|^{-1} \log n$, for some absolute constant $c>0$. That proof determined the best possible dependence of $k$ on $n$. The dependence of $k$ on $\varepsilon$ was improved by Gordon [7], who discovered another proof of Dvoretzky's theorem with $k(\varepsilon, n) \geq c \varepsilon^{2} \log n$. Both proofs are probabilistic; they show that not only do there exist almost Euclidean sections, but actually most sections are such. More precisely, if $X$ is a random subspace whose dimension does not exceed $k(\varepsilon, n)$, then the probability that the section $X \cap K$ is $(1+\varepsilon)$-Euclidean (common terminology for expressing that (7) holds) tends to 1 as $n \rightarrow \infty$. These facts motivate an investigation of the random $f$-vector $\{f(j, k, n)\}_{j=0}^{k-1}$, especially since it is well known that every $k$-dimensional symmetric polytope that has $2 n$ facets is affinely equivalent to a $k$-section of an $n$-cube.

\section{Vertices}

Let $G_{n, k}$ denote the set of $k$-dimensional subspaces of $\mathbb{R}^{n}$. We denote its normalized rotation invariant measure by "Prob." Recall that this measure is related to the normalized Haar measure $H$ of the orthogonal group $\mathrm{O}(n)$ by the equality

$$
\operatorname{Prob}\{X \in B\}=H\left\{g \in \mathrm{O}(n): g\left[e_{i}\right]_{i=1}^{k} \in B\right\},
$$


where $B$ is a Borel subset of $G_{n, k}$ and $\left[e_{i}\right]_{i=1}^{k}$ is the $k$-dimensional subspace spanned by the first $k$ unit vectors in $\mathbb{R}^{n}$. Fix $X \in G_{n, k}$. For each $0 \leq j \leq k-1$, the set of $j$-faces of the polytope $X \cap B_{\infty}^{n}$ coincides with the set of intersections of $(n-k+j)$-faces of $B_{\infty}^{n}$ with $X$. Every $(n-k+j)$-face of $B_{\infty}^{n}$ has the same probability to be intersected. Therefore if one particular $(n-k+j)$-face $F_{n-k+j}$ is fixed, then the expected number of $j$-faces of the section $X \cap B_{\infty}^{n}$ is equal to

$$
2^{k-j}\left(\begin{array}{c}
n \\
k-j
\end{array}\right) \operatorname{Prob}\left\{X \cap F_{n-k+j} \neq \emptyset\right\} .
$$

Let $C\left(F_{n-k+j}\right)$ denote the cone generated by $F_{n-k+j}$ :

$$
C\left(F_{n-k+j}\right)=\bigcup_{x \in F_{n-k+j}}\{t x: t \geq 0\} .
$$

Put $C_{1}\left(F_{n-k+j}\right)=C\left(F_{n-k+j}\right) \cap \mathbb{S}^{n-1}$. For every subspace $X$,

$$
X \cap F_{n-k+j} \neq \emptyset \quad \Longleftrightarrow \quad\left(X \cap \mathbb{S}^{n-1}\right) \cap C_{1}\left(F_{n-k+j}\right) \neq \emptyset .
$$

For $n=0,1, \ldots$ we denote by $\sigma_{n}$ the normalized rotation-invariant measure on the unit-sphere $\mathbb{S}^{n}$ in $\mathbb{R}^{n+1}$. The next lemma will prove useful for dealing with intersections of subsets of the sphere with random subspaces.

Lemma 2.1. Let $l, m, n$ be positive integers satisfying $l+m \geq n-1$. Suppose that $A \subset \mathbb{S}^{m}$ and $B \subset \mathbb{S}^{l}$ are Borel subsets. Then, for $p=l+m-n+1$,

$$
\int_{\mathrm{O}(n)} \sigma_{p}(g B \cap A) d H(g)=\sigma_{l}(B) \sigma_{m}(A) .
$$

To prove the lemma one observes that for fixed $A$ (resp. $B$ ) the integral defines an invariant measure on $\mathbb{S}^{l}$ (resp. $\mathbb{S}^{m}$ ); the conclusion follows from that.

Lemma 2.1 is now applied to $B=X \cap \mathbb{S}^{n-1}$, which we denote by $\mathbb{S}^{k-1}$, and to $A=C_{1}\left(F_{n-k+j}\right)$. For $l=k-1$ and $m=n-k+j$ equality (8) becomes

$$
\int_{\mathrm{O}(n)} \sigma_{j}\left(g \mathbb{S}^{k-1} \cap A\right) d H(g)=\sigma_{n-k+j}(A) .
$$

We are ready to compute the expected number of vertices. The Gaussian measure in $\mathbb{R}^{m}$ whose density is $(2 \pi)^{-m / 2} \exp \left(-\sum_{1}^{m} x_{i}^{2} / 2\right)$ is denoted by $\gamma_{m}$.

Proposition 2.2. The expected number of vertices of a random $k$-dimensional central section of the n-cube is given by the formula

$$
f(0, k, n)=2^{k}\left(\begin{array}{l}
n \\
k
\end{array}\right) \sqrt{\frac{2 k}{\pi}} \int_{0}^{\infty} e^{-k t^{2} / 2} \gamma_{n-k}\left(t B_{\infty}^{n-k}\right) d t .
$$

Proof. For each $g \in \mathrm{O}(n)$ we have

$$
g \mathbb{S}^{k-1} \cap C_{1}\left(F_{n-k}\right)=\left(\operatorname{span}\left(g \mathbb{S}^{k-1}\right) \cap C\left(F_{n-k}\right)\right) \cap \mathbb{S}^{n-1} .
$$


For almost every $g$ the intersection $\operatorname{span}\left(g \mathbb{S}^{k-1}\right) \cap C\left(F_{n-k}\right)$ is either the origin itself, or else a ray emanating from the origin. Therefore the intersection $g \mathbb{S}^{k-1} \cap C_{1}\left(F_{n-k}\right)$ is either empty or a singleton, for almost every $g$. Choose $j=0$ in (9), with $A=C_{1}\left(F_{n-k}\right)$. Since the measure $\sigma_{0}$ is concentrated on two points giving mass $\frac{1}{2}$ to each, we deduce from (9) that

$$
\operatorname{Prob}\left\{X \cap F_{n-k} \neq \emptyset\right\}=2 \sigma_{n-k}\left(C_{1}\left(F_{n-k}\right)\right) .
$$

To compute the right-hand side of (10), consider an $(n-k)$-dimensional cube of edgelength 1 inside $\mathbb{R}^{n-k+1}$, at a distance $\sqrt{k}$ from the origin, form the cone it generates, and compute the measure of its intersection with the sphere $\mathbb{S}^{n-k}$. Invoking polar coordinates we see that

$$
\sigma_{n-k}\left(C_{1}\left(F_{n-k}\right)\right)=\gamma_{n-k+1}\left(C\left(F_{n-k}\right)\right) .
$$

By rotational symmetry of the Gaussian measure we may assume that $F_{n-k}$ is specifically the set $\left\{x:\left|x_{i}\right| \leq 1,1 \leq i \leq n-k, x_{n-k+1}=\sqrt{k}\right\}$. The intersection of the hyperplane $\left\{x_{n-k+1}=t\right\}$ with $C\left(F_{n-k}\right)$ is an $(n-k)$-dimensional cube of edge-length $t / \sqrt{k}$. Therefore by Fubini's theorem

$$
\begin{aligned}
\gamma_{n-k+1}\left(C\left(F_{n-k}\right)\right) & =\frac{1}{\sqrt{2 \pi}} \int_{0}^{\infty} e^{-t^{2} / 2} \gamma_{n-k}\left(\frac{t}{\sqrt{k}} B_{\infty}^{n-k}\right) d t \\
& =\sqrt{\frac{k}{2 \pi}} \int_{0}^{\infty} e^{-k t^{2} / 2} \gamma_{n-k}\left(t B_{\infty}^{n-k}\right) d t
\end{aligned}
$$

The last equality, together with (10), implies the desired formula.

The next lemma points out the precise asymptotic behavior of $f(0, k, n)$ when $k$ is fixed and $n \rightarrow \infty$, and also that of $f(n-m, n-l, n)$, when $l, m$ are fixed and $n \rightarrow \infty$. (To be used in Section 3.)

Lemma 2.3. Suppose that $\left\{\alpha_{n}\right\}_{n=1}^{\infty}$ is a sequence of real numbers that has a positive limit $\alpha$. Then, as $n \rightarrow \infty$,

$$
\int_{0}^{\infty} e^{-\alpha_{n} t^{2} / 2} \gamma_{n}\left(t B_{\infty}^{n}\right) d t \sim \Gamma(\alpha) \frac{\pi^{\alpha / 2}}{\sqrt{2}} \frac{(\log n)^{\left(\alpha_{n}-1\right) / 2}}{n^{\alpha_{n}}},
$$

where $\Gamma$ is the gamma function.

Proof. Let $F_{n}(t)=\operatorname{Prob}\left\{\max _{i}\left|g_{i}\right| \leq t\right\}$, where $g_{1}, \ldots, g_{n}$ are independent $N(0,1)$ Gaussian variables. We have

$$
\gamma_{n}\left(t B_{\infty}^{n}\right)=\left(\sqrt{\frac{2}{\pi}} \int_{0}^{t} e^{-x^{2} / 2} d x\right)^{n}=F_{n}(t) .
$$

For $n>1$, put

$$
a_{n}=\frac{1}{\sqrt{2 \log n}} \quad \text { and } \quad b_{n}=\sqrt{2 \log n}-\frac{\log (\pi \log n)}{2 \sqrt{2 \log n}} .
$$


The well known tail approximation

$$
\sqrt{\frac{2}{\pi}} \int_{t}^{\infty} e^{-x^{2} / 2} d x=\sqrt{\frac{2}{\pi}} \frac{1+o(1)}{t} e^{-t^{2} / 2} \quad \text { as } \quad t \rightarrow \infty,
$$

combined with a simple calculation, implies that

$$
\lim _{n \rightarrow \infty} F_{n}\left(a_{n} x+b_{n}\right)=\exp \left(-e^{-x}\right), \quad \forall x \in \mathbb{R} .
$$

A change of variables gives

$$
\begin{aligned}
\int_{0}^{\infty} e^{-\alpha_{n} t^{2} / 2} \gamma_{n}\left(t B_{\infty}^{n}\right) d t= & a_{n} \int_{-b_{n} / a_{n}}^{\infty} e^{-\alpha_{n}\left(a_{n} x+b_{n}\right)^{2} / 2} F_{n}\left(a_{n} x+b_{n}\right) d x \\
= & \frac{\pi^{\alpha_{n} / 2}}{\sqrt{2}} \frac{(\log n)^{\left(\alpha_{n}-1\right) / 2}}{n^{\alpha_{n}}} e^{-o(1)} \\
& \cdot \int_{-\infty}^{\infty} e^{-x^{2} o(1)} e^{-\alpha_{n} x(1-o(1))} F_{n}\left(a_{n} x+b_{n}\right) \chi_{n}(x) d x .
\end{aligned}
$$

Here $\chi_{n}$ stands for the characteristic function of the interval $\left[-b_{n} / a_{n}, \infty\right)$. All four terms of the integrand in the last integral are nonnegative for each $x$. For $x \geq 0$ and sufficiently large $n$ we have $e^{-\alpha_{n} x(1-o(1))}<e^{-\alpha x / 2}$, while the rest of the terms are majorized by 1 . For $x<0$, the tail estimate (12) implies the existence of a constant $c=c(\alpha)>0$, such that $F_{n}\left(a_{n} x+b_{n}\right)<e^{(\alpha+1) x}$ for every $x$ in $(-\log n,-c)$, and sufficiently large $n$. One more application of (12) shows that $\lim _{n \rightarrow \infty} \int_{-b_{n} / a_{n}}^{-\log n} e^{-\alpha_{n} x} F_{n}\left(a_{n} x+b_{n}\right) d x=0$. By (13), the integrand converges pointwise to the function $e^{-\alpha x} \exp \left(-e^{-x}\right)$; Lebesgue's bounded convergence theorem can be applied:

$$
\begin{aligned}
\lim _{n \rightarrow \infty} \int_{-b_{n} / a_{n}} e^{-x^{2} o(1)} e^{-\alpha_{n} x(1-o(1))} F_{n}\left(a_{n} x+b_{n}\right) d x & =\int_{-\infty}^{\infty} e^{-\alpha x} \exp \left(-e^{-x}\right) d x \\
& =\Gamma(\alpha) .
\end{aligned}
$$

The proof of Lemma 2.3 is complete.

Taking $\alpha_{n} \equiv k$ in Lemma 2.3 and bearing in mind Proposition 2.2 re-proves the following result, which was mentioned in the Introduction.

Corollary 2.4. For fixed $k$,

$$
f(0, k, n) \sim \frac{2^{k}}{\sqrt{k}}(\pi \log n)^{(k-1) / 2} \quad \text { as } n \rightarrow \infty .
$$

We turn now to the case of fixed codimension. The next result is deduced from Proposition 2.2.

Proposition 2.5. For $d \geq 1$,

$$
f(0, n-d, n) \geq\left(\begin{array}{l}
n \\
d
\end{array}\right) 2^{n}\left(\frac{1}{\pi} \arctan \frac{1}{\sqrt{n-d}}\right)^{d} \quad(d \geq 1) .
$$


Equality holds for $d=1$ :

$$
f(0, n-1, n)=\frac{2^{n} n}{\pi} \arctan \frac{1}{\sqrt{n-1}} .
$$

Proof. Consider the probability measure $d \mu(t)=2 \sqrt{(k / \pi)} e^{-k t^{2}} d t$ on the half-line $[0, \infty)$. Put

$$
\Phi(t)=\frac{2}{\sqrt{\pi}} \int_{0}^{t} e^{-x^{2}} d x
$$

Then

$$
\gamma_{n-k}\left(t B_{\infty}^{n-k}\right)=\left(\sqrt{\frac{2}{\pi}} \int_{0}^{t} e^{-x^{2} / 2} d x\right)^{n-k}=\Phi^{n-k}\left(\frac{t}{\sqrt{2}}\right)
$$

Therefore

$$
\begin{aligned}
\sqrt{\frac{2 k}{\pi}} \int_{0}^{\infty} e^{-k t^{2} / 2} \gamma_{n-k}\left(t B_{\infty}^{n-k}\right) d t & =\sqrt{\frac{2 k}{\pi}} \int_{0}^{\infty} e^{-k t^{2} / 2} \Phi^{n-k}\left(\frac{t}{\sqrt{2}}\right) d t \\
& =\int_{0}^{\infty} \Phi^{n-k}(t) d \mu(t) \\
& \geq\left(\int_{0}^{\infty} \Phi(t) d \mu(t)\right)^{n-k} .
\end{aligned}
$$

Elementary calculation shows that

$$
\int_{0}^{\infty} e^{-k t^{2}} \Phi(t) d t=\frac{1}{\sqrt{\pi k}} \arctan \frac{1}{\sqrt{k}} .
$$

A combination of (15) with Proposition 2.2 gives the desired inequality, after a replacement of $k$ by $n-d$. Observe that, for $k=n-1$ (that is, $d=1$ ), there is equality in the inequality of (15).

Remarks. $\quad 1$. For $n=3$ we get, from $(14), f(0,2,3)=(24 / \pi) \arctan (1 / \sqrt{2}) \approx 4.7$. Therefore a random 2 -section of the 3 -cube is more likely to be a parallelogram than a hexagon.

2. Bárány and Lovász proved in [3] that (in particular) almost every $k$-section of the $n$-cube has at least $2^{k}$ vertices. Clearly this is a precise lower bound. For $k=n-1$, our result shows that the expected value is asymptotically $\sqrt{n} / \pi$ times the minimal value.

3. The asymptotic behavior of the integral

$$
\int_{0}^{\infty} e^{-k t^{2} / 2} \gamma_{n-k}\left(t B_{\infty}^{n-k}\right) d t
$$

for fixed $k$ and $n \rightarrow \infty$ was determined in [5] (following [9]), and was used to prove formula (4) of the Introduction. See also [1]. The asymptotic result is basically a corollary of the classical tail approximation of a single $N(0,1)$-Gaussian variable. Our approach to the proof of Lemma 2.3 seems to simplify the analysis. 
4. As was indicated in the Introduction, we can choose $\varepsilon=c / \sqrt{\log n}$ for some constant $c>0$, and then with high probability a random 2-section of the cube is $(1+c / \sqrt{\log n})$ Euclidean. It is well known that among all centrally symmetric polygons having $2 m$ vertices, the regular $2 m$-gon minimizes the Banach-Mazur distance to the Euclidean disk; the minimal distance is $(\cos (\pi / 2 m))^{-1}$. Consequently with high probability we have

$$
\left(\cos \frac{\pi}{2 m}\right)^{-1}<1+\frac{c}{\sqrt{\log n}}
$$

Hence most 2-sections of the $n$-cube have at least $C(\log n)^{1 / 4}$ vertices, for some positive constant $C$. By Proposition 2.2 (after suitable rearrangement)

$$
f(0,2, n)=2 \sqrt{\pi} \mathbf{E}\left(\max _{1 \leq i \leq n}\left|g_{i}\right|\right),
$$

which is of the order of magnitude of $\sqrt{\log n}$. Summarizing these observations, we conclude: a typical 2-section of the $n$-cube is $(1+c / \sqrt{\log n})$-Euclidean, hence it cannot have too few vertices - it has at least $C(\log n)^{1 / 4}$ vertices with probability that tends to 1 as $n \rightarrow \infty$. It does not however tend to be a regular polygon, because the expected number of its vertices is too high for that.

\section{Other Faces}

We now turn to the case $j \geq 1$, and prove the following result.

Proposition 3.1. For $j \geq 1$, the following inequality holds:

$$
\frac{f(0, k-j, n)}{f(j, k, n)}<\sqrt{\frac{2}{\pi}}\left(\frac{j(k-j)}{n-k+j}\right)^{1 / 2} \int_{0}^{\infty} \exp \left(-\frac{j(k-j)}{n-k+j} t^{2} / 2\right) \gamma_{j}\left(t B_{\infty}^{j}\right) d t .
$$

The starting point in the proof of Proposition 3.1 is (9). Again, we choose $A=C_{1}\left(F_{n-k+j}\right)$. The random variable $g \rightarrow \sigma_{j}\left(g \mathbb{S}^{k-1} \cap A\right)$, which is defined on $\mathrm{O}(n)$, has values in $[0,1]$. Hence

$$
\int_{\mathrm{O}(n)} \sigma_{j}\left(g \mathbb{S}^{k-1} \cap A\right) d H(g)=\int_{0}^{1} H\left\{g: \sigma_{j}\left(g \mathbb{S}^{k-1} \cap A\right) \geq t\right\} d t .
$$

The integrand is nonincreasing, and

$$
H\left\{g: \sigma_{j}\left(g \mathbb{S}^{k-1} \cap A\right) \geq 0\right\}=\operatorname{Prob}\left\{X \cap F_{n-k+j} \neq \emptyset\right\},
$$

because the event $\left\{g \mathbb{S}^{k-1} \cap A \neq \emptyset\right.$ and $\left.\sigma_{j}\left(g \mathbb{S}^{k-1} \cap A\right)=0\right\}$ has Haar measure zero. Therefore, by (9),

$$
\begin{aligned}
\sigma_{n-k+j}(A) & \leq \operatorname{Prob}\left\{X \cap F_{n-k+j} \neq \emptyset\right\} \sup \left\{t: H\left\{g: \sigma_{j}\left(g \mathbb{S}^{k-1} \cap A\right) \geq t\right\}>0\right\} \\
& \leq \operatorname{Prob}\left\{X \cap F_{n-k+j} \neq \emptyset\right\} \sup \left\{\sigma_{j}\left(g \mathbb{S}^{k-1} \cap A\right): g \in \mathrm{O}(n)\right\} .
\end{aligned}
$$


Let

$$
t_{j, k, n}=\sup \left\{\sigma_{j}\left(g \mathbb{S}^{k-1} \cap A\right): g \in \mathrm{O}(n)\right\} .
$$

By (9), (15), and (16) we get

$$
\operatorname{Prob}\left\{X \cap F_{n-k+j} \neq \emptyset\right\} \geq \frac{\sigma_{n-k+j}(A)}{t_{j, k, n}} .
$$

Hence, by (10),

$$
f(j, k, n) \geq 2^{k-j}\left(\begin{array}{c}
n \\
k-j
\end{array}\right) \frac{\sigma_{n-k+j}(A)}{t_{j, k, n}}=\frac{\frac{1}{2} f(0, k-j, n)}{t_{j, k, n}} .
$$

We must bound $t_{j, k, n}$ from above. Since $A$ is contained in a half-space, a trivial bound is $t_{j, k, n} \leq \frac{1}{2}$. In some cases this bound can be significantly improved. The main lemma in this section is the following.

Lemma 3.2. If $1 \leq j<k<n$, then

$$
t_{j, k, n} \leq \frac{1}{\sqrt{2 \pi}}\left(\frac{j(k-j)}{n-k+j}\right)^{1 / 2} \int_{0}^{\infty} \exp \left(-\frac{j(k-j)}{n-k+j} t^{2} / 2\right) \gamma_{j}\left(t B_{\infty}^{j}\right) d t
$$

The next lemma will be used in the proof of Lemma 3.2.

Lemma 3.3. Given a positive number $\tau>0, a j$-dimensional affine subspace $Y$ of $\mathbb{R}^{m}$, and a point $y_{0} \in Y$, the following inequality holds:

$$
\gamma_{j}\left(\left(Y \cap \tau B_{\infty}^{m}\right)-y_{0}\right) \leq \gamma_{j}\left(\tau \sqrt{\frac{m}{j}} B_{\infty}^{j}\right) .
$$

Proof. Let $Q$ denote the orthogonal projection onto $Y-y_{0}$. As usual, $\left\{e_{i}\right\}_{i=1}^{m}$ are the standard unit vectors in $\mathbb{R}^{m}$. Put $u_{i}=Q e_{i} /\left\|Q e_{i}\right\|$ if $Q e_{i} \neq 0$, and $u_{i}=0$ otherwise; put $c_{i}=\left\|Q e_{i}\right\|^{2}$ and $\alpha_{i}=\left\langle y_{0}, e_{i}\right\rangle$ for $1 \leq i \leq m .(\langle\cdot, \cdot\rangle$ is the standard scalar product.) Then

$$
\begin{aligned}
Y \cap \tau B_{\infty}^{m} & =\left\{y \in Y:\left|\left\langle y, e_{i}\right\rangle\right| \leq \tau, \quad \forall i\right\} \\
& =\left\{y \in Y:\left|\left\langle y-y_{0}, e_{i}\right\rangle+\left\langle y_{0}, e_{i}\right\rangle\right| \leq \tau, \quad \forall i\right\} \\
& =\left\{y \in Y: \frac{-\alpha_{i}-\tau}{\sqrt{c_{i}}} \leq\left\langle y-y_{0}, u_{i}\right\rangle \leq \frac{-\alpha_{i}+\tau}{\sqrt{c_{i}}}\right\} .
\end{aligned}
$$

Therefore

$$
\left(Y \cap \tau B_{\infty}^{m}\right)-y_{0}=\left\{x \in Y-y_{0}: \frac{-\alpha_{i}-\tau}{\sqrt{c_{i}}} \leq\left\langle x, u_{i}\right\rangle \leq \frac{-\alpha_{i}+\tau}{\sqrt{c_{i}}}\right\} .
$$

Now we can imitate Ball's argument from [2] concerning sections of maximal volume. Instead of the Lebesgue measure, we have to consider the Gaussian measure. 
In $Y-y_{0}$, the identity operator can be written as $\sum_{1}^{m} c_{i} u_{i} \otimes u_{i}$. In particular,

$$
\sum_{i=1}^{m} c_{i}=j \quad \text { and } \quad\|x\|^{2}=\sum_{i=1}^{m} c_{i}\left\langle x, u_{i}\right\rangle^{2}, \quad \forall x \in Y-y_{0} .
$$

Therefore the Gaussian measure in $Y-y_{0}$ is equal to

$$
(2 \pi)^{-j / 2} \exp \left(-\frac{1}{2} \sum_{i=1}^{m} c_{i}\left\langle x, u_{i}\right\rangle^{2}\right) d x .
$$

Let $\chi_{i}$ denote the characteristic function of the interval $\left[\left(-\alpha_{i}-\tau\right) / \sqrt{c_{i}},\left(-\alpha_{i}+\tau\right) / \sqrt{c_{i}}\right]$. Then, by the above,

$$
\begin{aligned}
\gamma_{j}\left(\left(Y \cap \tau B_{\infty}^{m}\right)-y_{0}\right) & =(2 \pi)^{-j / 2} \int_{Y-y_{0}}\left(\prod_{i=1}^{m} \chi_{i}\left(\left\langle x, u_{i}\right\rangle\right) e^{-c_{i}\left\langle x, u_{i}\right\rangle^{2} / 2}\right) d x \\
& =(2 \pi)^{-j / 2} \int_{Y-y_{0}} \prod_{i=1}^{m}\left(\chi_{i}\left(\left\langle x, u_{i}\right\rangle\right) e^{-\left\langle x, u_{i}\right\rangle^{2} / 2}\right)^{c_{i}} d x \\
& \leq(2 \pi)^{-j / 2} \prod_{i=1}^{m}\left(\int_{\left(-\alpha_{i}-\tau\right) / \sqrt{c_{i}}}^{\left(-\alpha_{i}+\tau\right) / \sqrt{c_{i}}} e^{-s^{2} / 2} d s\right)^{c_{i}} .
\end{aligned}
$$

The last inequality is a consequence of Brascamp-Lieb's inequality, which is stated in [2] as follows:

Lemma. Let $\left(u_{i}\right)_{1}^{m}$ be a sequence of unit vectors in $\mathbb{R}^{n}$ and let $\left(c_{i}\right)_{1}^{m}$ be a sequence of positive numbers so that

$$
\sum_{1}^{m} c_{i} u_{i} \otimes u_{i}=I_{n}
$$

For each $i$, let $f_{i}: \mathbb{R} \rightarrow[0, \infty)$ be integrable. Then

$$
\int_{\mathbb{R}^{n}} \prod_{i=1}^{m} f_{i}\left(\left\langle u_{i}, x\right\rangle\right)^{c_{i}} d x \leq \prod_{i=1}^{m}\left(\int_{\mathbb{R}} f_{i}\right)^{c_{i}} .
$$

The $i$ th integral in the product of (19) is not larger than $\int_{-\tau / \sqrt{c_{i}}}^{\tau / \sqrt{c_{i}}} e^{-s^{2} / 2} d s$. Hence the last expression in (19) is bounded above by

$$
(2 \pi)^{-j / 2} \prod_{i=1}^{m}\left(2 \int_{0}^{\tau / \sqrt{c_{i}}} e^{-s^{2} / 2} d s\right)^{c_{i}},
$$

which is maximized when all the $c_{i}$ 's are equal. Hence

$$
\begin{aligned}
\gamma_{j}\left(\left(Y \cap \tau B_{\infty}^{m}\right)-y_{0}\right) & \leq\left(\sqrt{\frac{2}{\pi}} \int_{0}^{\tau \sqrt{m / j}} e^{-s^{2} / 2} d s\right)^{j} \\
& =\gamma_{j}\left(\tau \sqrt{\frac{m}{j}} B_{\infty}^{j}\right) .
\end{aligned}
$$

The proof of Lemma 3.3 is complete. 
Proof of Lemma 3.2. For $g \in \mathrm{O}(n)$,

$$
\begin{aligned}
\sigma_{j}\left(g \mathbb{S}^{k-1} \cap A\right) & =\gamma_{j+1}\left(C\left(F_{n-k+j}\right) \cap \operatorname{span}\left(g \mathbb{S}^{k-1}\right)\right) \\
& =\gamma_{j+1}\left(C\left[F_{n-k+j} \cap \operatorname{span}\left(g \mathbb{S}^{k-1}\right)\right]\right) .
\end{aligned}
$$

The second equality is a consequence of the identity $C\left(F_{n-k+j}\right) \cap X=C\left(F_{n-k+j} \cap X\right)$, which trivially holds for every subspace $X \subset \mathbb{R}^{n}$. Fix a subspace $X \in G_{n, k}$ for which the section $X \cap F_{n-k+j}$ is $j$-dimensional; almost every $X \in G_{n, k}$ has this property. Let $C$ denote the $(j+1)$-dimensional cone generated by $X \cap F_{n-k+j}$; put $X_{0}=\operatorname{span} C$. By $M$ we denote the affine subspace spanned by $X \cap F_{n-k+j}$, and by $d$ its distance from the origin of $X$. The Gaussian measure of the cone $C$ is computed as follows. Take the unit vector $\xi \in X_{0}$ which is orthogonal to $M$, and for which $d \xi \in M$. For $t>0$, put $W_{t}=\left\{x \in X_{0}:\langle x, \xi\rangle=t\right\}$. Observe that $C \cap W_{t}=(t / d)\left(X \cap F_{n-k+j}\right)$. Let $P$ denote the orthogonal projection from $X_{0}$ onto $W_{0}$. By Fubini's theorem,

$$
\begin{aligned}
\gamma_{j+1}(C) & =\frac{1}{\sqrt{2 \pi}} \int_{0}^{\infty} e^{-t^{2} / 2} \gamma_{j}\left(P\left(C \cap W_{t}\right)\right) d t \\
& =\frac{1}{\sqrt{2 \pi}} \int_{0}^{\infty} e^{-t^{2} / 2} \gamma_{j}\left(P \frac{t}{d}\left(X \cap F_{n-k+j}\right)\right) d t
\end{aligned}
$$

Our task is to estimate the expression $\gamma_{j}\left(P \tau\left(X \cap F_{n-k+j}\right)\right)$ for every $\tau>0$. We need to discuss Gaussian measures in different subspaces. Whenever $M$ is an $m$-dimensional subspace of $\mathbb{R}^{n}$ and $q \in M$, let $\mathbb{G}_{M, q}$ denote the measure $(2 \pi)^{-m / 2} \exp \left(-\|x-q\|^{2} / 2\right) d x$. In case $M$ is an $m$-dimensional linear subspace of $\mathbb{R}^{n}$ and $q=0$ we simply write $\mathbb{G}_{M, 0}=\gamma_{m}$. If $T$ is an isometry of $\mathbb{R}^{n}$, then for every Borel subset $S \subset M$ we have

$$
\mathbb{G}_{M, q}(S)=\mathbb{G}_{T M, T q}(T S) .
$$

We momentarily assume that $\tau=1$. Let $q$ denote the nearest point of $M$ to the origin of $X$. Both $M$ and the range of the projection $P$ are $j$-dimensional affine subspaces of $X_{0}$. We have

$$
P\left(X \cap F_{n-k+j}\right)=\left(X \cap F_{n-k+j}\right)-q,
$$

hence, by (22),

$$
\mathbb{G}_{M, q}\left(X \cap F_{n-k+j}\right)=\gamma_{j}\left(P\left(X \cap F_{n-k+j}\right)\right) .
$$

Now let $L$ denote the affine subspace spanned by $F_{n-k+j}$, whose origin $O_{L}$ is taken as the center of the face $F_{n-k+j}$. (So if $X$ passes through the center of $F_{n-k+j}$, then $q=O_{L}$.) $M$ is also a $j$-dimensional affine subspace of $L$. By (22),

$$
\mathbb{G}_{M, q}\left(X \cap F_{n-k+j}\right)=\mathbb{G}_{M-\left(q-O_{L}\right), O_{L}}\left(\left(X \cap F_{n-k+j}\right)-\left(q-O_{L}\right)\right) .
$$

Applying the same argument for arbitrary $\tau>0$ we conclude that

$$
\gamma_{j}\left(P \tau\left(X \cap F_{n-k+j}\right)\right)=\mathbb{G}_{\tau M-\tau\left(q-O_{L}\right), \tau O_{L}}\left(\tau\left(X \cap F_{n-k+j}\right)-\tau\left(q-O_{L}\right)\right) .
$$

We may think of $\tau L$ as $\mathbb{R}^{n-k+j}$, of $\tau F_{n-k+j}$ as $\tau B_{\infty}^{n-k+j}$, and of $\tau\left(X \cap F_{n-k+j}\right)$ as an affine $j$-dimensional section of $\tau B_{\infty}^{n-k+j}$. Thus for each $t>0$ Lemma 3.3 can be 
used with $\tau=t / d$ and $m=n-k+j$. By the definition of $d$, we have $d \geq \sqrt{k-j}$. Combining (18), (21), and (24) we deduce that

$$
\begin{aligned}
\gamma_{j+1}(C) & \leq \frac{1}{\sqrt{2 \pi}} \int_{0}^{\infty} e^{-t^{2} / 2} \gamma_{j}\left(t\left(\frac{n-k+j}{j(k-j)}\right)^{1 / 2} B_{\infty}^{j}\right) d t \\
& =\frac{1}{\sqrt{2 \pi}}\left(\frac{j(k-j)}{n-k+j}\right)^{1 / 2} \int_{0}^{\infty} \exp \left(-\frac{j(k-j)}{n-k+j} t^{2} / 2\right) \gamma_{j}\left(t B_{\infty}^{j}\right) d t .
\end{aligned}
$$

The proof of Lemma 3.2 and thus of Proposition 3.1 is complete.

By using the asymptotic formulas of Section 2, namely Lemma 2.3 and Corollary 2.4, we can now prove the following result, which shows that the lower bound for $f(j, k, n)$ derived from Proposition 3.1 is, in some cases, asymptotically best possible.

Corollary 3.4. For fixed integers $1 \leq l<m$,

$$
f(n-m, n-l, n) \backsim \frac{(2 n)^{m-l}}{(m-l) !} \quad \text { as } \quad n \rightarrow \infty .
$$

Proof. Put $\alpha_{n}=(m-l)(n-m) /(n-m+l)$. By Proposition 3.1,

$$
\frac{f(0, m-l, n)}{f(n-m, n-l, n)}<\sqrt{\frac{2 \alpha_{n}}{\pi}} \int_{0}^{\infty} e^{-\alpha_{n} t^{2} / 2} \gamma_{n-m}\left(t B_{\infty}^{n-m}\right) d t .
$$

Put $b_{n}=(\log (n-m))^{\left(\alpha_{n}-1\right) / 2} /(n-m)^{\alpha_{n}}$ and $c_{n}=(\log n)^{(m-l-1) / 2}$. Let $d_{n}$ denote the right-hand side of (26), from which we get

$$
f(n-m, n-l, n) \frac{b_{n}}{c_{n}}>\frac{f(0, m-l, n)}{c_{n}} \frac{b_{n}}{d_{n}} .
$$

Since $\lim _{n \rightarrow \infty} \alpha_{n}=(m-l)$, Lemma 2.3 implies that

$$
\lim _{n \rightarrow \infty} \frac{b_{n}}{d_{n}}=\frac{1}{\pi^{(m-l-1) / 2} \Gamma(m-l) \sqrt{m-l}} .
$$

Moreover, by Corollary 2.4,

$$
\lim _{n \rightarrow \infty} \frac{f(0, m-l, n)}{c_{n}}=\frac{2^{m-l} \pi^{(m-l-1) / 2}}{\sqrt{m-l}} .
$$

Thus, the sequence $f(n-m, n-l, n)\left(b_{n} / c_{n}\right)$ is larger than a sequence that tends to $2^{m-l} /(m-l)$ ! as $n$ tends to infinity. On the other hand we have $f(n-m, n-l, n)<$ $2^{m-l}\left(\begin{array}{c}n \\ m-l\end{array}\right)$, so

$$
f(n-m, n-l, n) \frac{b_{n}}{c_{n}}<2^{m-l}\left(\begin{array}{c}
n \\
m-l
\end{array}\right) \frac{b_{n}}{c_{n}},
$$

and since $b_{n} / c_{n} \backsim n^{l-m}$, the right-hand side here tends to $2^{m-l} /(m-l)$ !. Consequently,

$$
\lim _{n \rightarrow \infty} f(n-m, n-l, n) \frac{b_{n}}{c_{n}}=\frac{2^{m-l}}{(m-l) !} .
$$


The required asymptotic formula follows immediately. The proof of Corollary 3.4 is complete.

Remarks. 1. The previous corollary implies that the number of $(n-m)$-faces of a random $(n-l)$-section of the $n$-cube tends to concentrate near the value $2^{m-l}\left(\begin{array}{c}n \\ m-l\end{array}\right)$, which bounds it from above. So, for example, a typical 1-codimensional section of the $n$-cube will have $2 n-o(n)$ facets as $n \rightarrow \infty$. This result can also be deduced from the identity (3). Indeed, by duality we have $f(n-m, n-l, n)=\mathbf{E}\left(f_{m-l-1}\left(\Pi_{n-l}\left(B_{1}^{n}\right)\right)\right)$, and replacing $T^{n}$ by $B_{1}^{n}$ in the proof of Theorem 2 in [1] (the details of this replacement appear in [5]; see the proof of Theorem 1.1 there) we get the previous corollary.

2. According to a remark made in [5], the number $f(j, k, n)$ is equal to the expected number of $(k-j-1)$-faces of the convex hull of $\pm G_{1}, \ldots, \pm G_{n}$, where the $G_{i}$ 's are independent copies of a $k$-dimensional Gaussian vector. See also [4]. Hence, the results for $f(0, k, n)$ can be interpreted as results for the expected number of facets of the convex hull of $\left\{ \pm G_{i}\right\}_{1}^{n}$ in $\mathbb{R}^{k}$. For example, we can translate the first remark at the end of Section 2 to the following statement:

If three points in the plane are chosen at random, then their symmetric convex hull is more likely to be a parallelogram than a hexagon.

\section{Acknowledgments}

I thank Itai Benjamini for getting me interested in the subject of this paper, and for several stimulating discussions. I also thank the referees for their useful comments.

\section{References}

1. F. Affentranger and R. Schneider, Random projections of regular simplices, Discrete Comput. Geom. 7 (1992), 219-226.

2. K. Ball, Volumes of Sections of Cubes and Related Problems, Lecture Notes in Mathematics (J. Lindenstruass and V. Milman, eds.), vol. 1376, Springer-Verlag, Berlin, 1989, pp. 251-260.

3. I. Bárány and L. Lovász, Borsuk's theorem and the number of facets of centrally symmetric polytopes, Acta Math. Acad. Sci. Hungar. 40(3-4) (1982), 323-329.

4. Y. M. Baryshnikov and R. A. Vitale, Regular simplices and Gaussian samples, Discrete Comput. Geom. 11(2) (1994), 141-147.

5. K. Böröczky, Jr., and M. Henk, Random projections of regular polytopes, Arch. Math., to appear.

6. T. Figiel, J. Lindenstrauss, and V. Milman, The dimension of almost spherical sections of convex bodies, Acta Math. 139 (1977), 53-94.

7. Y. Gordon, Some inequalities for Gaussian processes and applications, Israel J. Math. 50 (1985), 265-289.

8. B. Grünbaum, Convex Polytopes, Interscience, London, 1967.

9. H. Raynaud, Sur l'enveloppe convexe des nuages de points aleatoires $\mathbb{R}^{n}$, I, J. Appl. Probab. 7 (1970), 35-48.

10. A. M. Vershik and P. V. Sporyshev, Asymptotic behavior of the number of faces of random polyhedra and the neighborliness problem, Selecta Math. Soviet. 11(2) (1992), 181-201 (English translation).

Received August 1, 1998, and in revised form December 15, 1998. 